\title{
Size-dependent thermal conductivity of nanoscale semiconducting systems
}

\author{
L. H. Liang ${ }^{1}$ and Baowen $\mathrm{Li}^{1,2,3 *}$ \\ ${ }^{1}$ Department of Physics and Center for Computational Science and Engineering, \\ National University of Singapore, Singapore 117542, Republic of Singapore \\ ${ }^{2}$ Laboratory of Modern Acoustics and Institute of Acoustics, Nanjing University, 210093, PR China \\ ${ }^{3}$ NUS Graduate School for Integrative Sciences and Engineering, Singapore 117597, Republic of Singapore
}

(Dated: 6 March 2006)

\begin{abstract}
We study the size dependence of thermal conductivity in nanoscale semiconducting systems. An analytical formula including the surface scattering and the size confinement effects of phonon transport is derived. The theoretical formula gives good agreements with the existing experimental data for $\mathrm{Si}$ and GaAs nanowires and thin films.

PACS numbers: 68.65.-k, 65.80.+n, 66.70.+f, 44.10.+i
\end{abstract}

It is of great importance from both fundamental and application point of views to understand heat conduction in nanoscale materials. On one hand, many fundamental questions remain open, such as whether the thermodynamical laws still apply to the nanoscale materials which are characterized by finite number of atoms/molecules, while the thermodynamics is established on the fact that the system contains infinite large number of molecules. On the other hand, a continuous miniaturization and increase of running speed of semiconductor devices will give rise to redundant heat that will deteriorate the devices' efficiency $\frac{1}{1}$ How to dissipate the heat from these devices becomes a crucial issue in heat management.

Generally, two approaches are used in studying heat conduction in such low-dimensional systems of finite size. At first, in order to understand the physical mechanism of heat conduction in such systems, many one-dimensional (1D) lattice models with and without on-site (pinning) potential are used such as the Fermi-Pasta-Ulam (FPU) model ${ }^{2}$ without onsite potential, and the Frenkel Kontoroval (FK) model and the $\phi^{4}$ model with on-site potential ${ }^{\frac{3}{3}}$ More recently, a polymer model with transverse motion has been introduced and studied both analytically and numerically ${ }_{*}^{4}$ All these models nicely demonstrate the role of anharmonicity and the role of on-site potential in 1D heat conduction. They are useful in helping us understand the fundamental law of heat conduction, the Fourier law. In the system without on-site potential, a sizedependent thermal conductivity has been observed, 2 , which is attributed to a superdiffusive motion of phonons $\frac{\sqrt{5}}{}$ However, such models are too simple to be used for modelling heat conduction in realistic nanostructures, because the heat conduction in such models is restricted to $1 \mathrm{D}$ or quasi-1D, the scattering of phonons from surfaces is completely neglected.

Another popular approach employs the Boltzmann transport equation, which can explain the bulk transport phenomena well. However, as we know that for nanostructures, both the effects of phonon confinement and non-equilibrium phonon distribution due to boundary scattering are important. Indeed, recent theoretical and experimental investigation demonstrates a size-dependent behavior of lattice vibration of

\footnotetext{
${ }^{*}$ Correspondence should be addressed to: phylibw@ nus.edu.sg
}

nanostructures $\underline{\underline{6}}$ Experimental measurements also show that thermal conductivity of semiconducting nanowires and thin films is smaller than the corresponding bulk value ${ }^{7.89,10}$ However, a general satisfactory theory to give a prediction agreed with existing experimental results at room temperature is not available yet.

In this paper, we propose a phenomenological theory for the size dependence of thermal conductivity by taking into account the intrinsic size effect of phonon velocity, mean free path and the surface scattering effect. An explicit analytic expression is obtained, in which all parameters have clear physical meaning, and good agreements with the existing experiments are found. The different roles of two basic effects in the different size range are illustrated.

We start with the well-known kinetic formula of thermal conductivity $\kappa$ for bulk dielectric materials,, 11

$$
\kappa=\frac{1}{3} c v l,
$$

where $c$ is the specific heat, $v$ the average phonon velocity and $l$ the mean free path (MFP). We assume that phonons predominate the thermal conduction, which is true for semiconductors or insulators discussed here. For thermal conductivity of nanomaterials, we first consider the size dependence of $v$ and $l$. The specific heat is assumed to be constant and the room temperature is applied in this paper. The average phonon velocity is proportional to the characteristic Debye temperature of crystals, ${ }^{12,13}$

$$
\Theta \propto \frac{2 h}{\pi k_{B}}\left(\frac{3 N_{A}}{4 \pi V}\right)^{1 / 3} v
$$

with the Planck constant $h$, the Boltzmann constant $k_{B}$, the Avogadro constant $N_{A}$, and the molar volume $V$. The system considered is assumed to be isotropic.

In the following discussion, we suppose Eq. (2) is valid for the corresponding nanoscale crystals. Let $L$ be the size of nanostructures, such as the diameter of nanowires or the thickness of thin films, the $L \rightarrow \infty$ limit is denoted by using the subscript $b$ which means the corresponding bulk limit, the size dependence of the phonon velocity is equal to that of the Debye temperature, we thus have, $v_{L} / v_{b}=\Theta_{L} / \Theta_{b}$.

From the Lindemann's proposition, we may get the relationship between the melting point and the Debye temperature 
of crystals. In 1910, Lindemann proposed a melting criterion, known to be valid for small particles, $\frac{14}{14}$ stating that a crystal will melt when the root mean square displacement (MSD) $\sigma$ of atoms in the crystal exceeds a certain fraction of the interatomic distance 15 Combining with the Einstein specific heat theory, the square of the characteristic temperature is proportional to the melting point $T^{m}$ of crystals, and the modern form of this relation for the Debye temperature is ${ }^{11.16}$

$$
\Theta=\text { const. }\left(\frac{T^{m}}{M V^{2 / 3}}\right)^{1 / 2}
$$

with the molecular mass $M$. According to the same relation for nanocrystals,

$$
\Theta_{L}^{2} / \Theta_{b}^{2}=T_{L}^{m} / T_{b}^{m} .
$$

Combining the Lindemann melting formula and the Debye expression for thermal conductivity, eliminating the Debye temperature and the sound velocity, it can be deduced that the MFP is proportional to the melting point at a given absolute temperature $T, l \propto 20 T^{m} a /\left(\gamma^{2} T\right)$ with the lattice constant $a$ and the Gruneisen constant $\gamma, 11.17$ Similarly, the same relation is assumed for nanocrystals, we have

$$
l_{L} / l_{b}=T_{L}^{m} / T_{b}^{m} .
$$

From Eqs. (1), (2), (4) and (5), we obtain an expression for the size-dependent thermal conductivity of nanosemiconductors $\left[T_{L}^{m} / T_{b}^{m}\right]^{3 / 2}$. Moreover, based on the fact of the sizedependent atomic thermal vibrations, the relations among the atomic MSD $\sigma^{2}$, the Debye temperature and the melting point, the size-dependent melting temperature function of nanocrystals has been modelled and validated as follows 18

$$
T_{L}^{m} / T_{b}^{m}=\left(\sigma_{b} / \sigma_{L}\right)^{2}=\exp \left(\frac{-(\alpha-1)}{L / L_{0}-1}\right)
$$

where $\alpha\left(\equiv \sigma_{s}^{2} / \sigma_{i}^{2}\right)$ is a material constant with $\sigma_{s}^{2}$ and $\sigma_{i}^{2}$ corresponding to the MSD of surface atoms of a crystal and that of atoms within the crystal, respectively. For the free-standing nanocrystals, $\alpha=2 S_{v} /(3 R)+1>1$ with the bulk vibrational entropy $S_{v}$ of melting and the ideal gas constant $R^{18}$ based on the Mott's expression for the vibrational entropy and its relation with the melting point ${ }^{13,19} L_{0}$ is a critical size at which almost all atoms of a crystal are located on its surface, $L_{0}=2(3-d) w$ with the atomic/molecular diameter $w$ and the dimension $d=0,1,2$ for nanoparticles, nanowires and thin films, respectively 18 Note that the two basic assumptions in the model of the size-dependent atomic thermal vibrations of nanocrystals are: (1) although ${\sigma_{s}}^{2}$ and ${\sigma_{i}}^{2}$ are considered to be size-dependent (the phonon softening is considered to occur not only on the surface but also in the interior for small size crystals), $\alpha$, the ratio between them, is taken approximately as a size-independent value; (2) the cooperative coupling between the surface region and the interior region is considered phenomenologically by taking the variation of $\sigma^{2}$ to be dependent on the value of itself, $\sigma^{2}$ is the average MSD over the crystal with the respective weight of
TABLE I: Parameters used in Eq. (7) for predictions in this paper. $\alpha=2 S_{v} /(3 R)+1, S_{v}=S_{m}-R$ (Ref. 22) and $S_{m}=H_{m} / T^{m}$ with the bulk melting entropy $S_{m}$, enthalpy $H_{m}$ and temperature $T^{m} . L_{0}=4 w, 2 w$ for nanowires and thin films, respectively.

\begin{tabular}{|c|c|c|c|c|}
\hline & $H_{m}\left(\mathrm{KJmol}^{-1}\right)$ & $T_{m}(\mathrm{~K})$ & $w(\mathrm{~nm})$ & $l_{0}(\mathrm{~nm})$ \\
\hline $\mathrm{Si}$ & $50.55^{23}$ & $1685^{23}$ & $0.3368^{24}$ & $41^{25}$ \\
\hline $\mathrm{GaAs}$ & $120^{2 \underline{\underline{\underline{T}}}}$ & $1511^{23.26}$ & $0.245^{27}$ & $5.8^{28}$ \\
\hline
\end{tabular}

$\sigma_{s}{ }^{2}$ and $\sigma_{i}{ }^{2} \underline{18}$ With the above consideration, a change in $\sigma^{2}$ can be given by $\sigma^{2}(x+d x)-\sigma^{2}(x)=(\alpha-1) \sigma^{2}(x) d x$, where the surface-volume ratio $x=L_{0} /\left(L-L_{0}\right)$. By integrating the above equation, the size-dependent MSD was obtained as shown in Eq. (6). According to $\sigma^{2} \propto T / \Theta^{2}$ in the high temperature approximation $(T>\Theta / 2)^{16,20}$ and Eq. (4), $\sigma_{b}^{2} / \sigma_{L}^{2}=\Theta_{L}^{2} / \Theta_{b}^{2}=T_{L}^{m} / T_{b}^{m}$. Equation (6) indicates that the melting point and thus the thermal conductivity decreases with reducing size $L$ of nanocrystals, it is valid for crystals of $L \geq 2 L_{0}$ with $2 L_{0}$ representing a characteristic length scale for the crystallinity 18

The above discussion on the thermal conductivity of nanostructures, based on the effective bulk formula, includes the intrinsic size effect of the phonon velocity and the MFP. The phonon-phonon interaction increases with size reduction due to the confinement, which causes the increase of thermal resistance and the decrease of heat conduction. On the other hand, as the size decreases and the surface-volume ratio increases, the large surface/interface scattering, corresponding to certain boundary conditions in the linearized Boltzmann equation, has great influence on the transport. Considering the nonequilibrium phonon distribution due to boundary scattering, the effect of the surface roughness with the boundary scattering shows an exponential suppression in the distribution and the conduction. ${ }^{11,21}$ Therefore, a term $p \exp \left(-l_{0} / L\right)$ is added to correct the bulk formula, where $p$ is a factor reflecting the surface roughness, $l_{0}$ is the phonon MFP in the Debye model at room temperature and assumed to be a constant here since we have considered the size effect in the above discussion. $l_{0} / L$ corresponds to the Knudsen number of the phonon Knudsen flow induced by the interface scattering ${ }^{21}$ Finally, the size-dependent thermal conductivity is obtained as

$$
\frac{\kappa_{L}}{\kappa_{b}}=p \exp \left(-\frac{l_{0}}{L}\right)\left[\exp \left(\frac{-(\alpha-1)}{L / L_{0}-1}\right)\right]^{3 / 2} \text {. }
$$

This is the central result of this paper. It gives a quantitative prediction for the size-dependent thermal conductivity. $0<p \leq 1$. The larger value of $p$ corresponds to the smaller roughness, i.e. the smoother surface, thus the more probability of specular scattering, vice versa, the smaller $p$ corresponds to the more probability of diffusive scattering. The smaller $L$ corresponds to the relatively larger surface roughness usually and thus the smaller $p . p$ depends greatly on fabrication precision of nanostructures, its physical meaning will be discussed further later on. When system size deceases, the value of $\exp \left(-l_{0} / L\right)$ decreases, which reflects the increase of the interface scattering and thus the weakened conduction. From the point of view of the bulk approach, there are two 


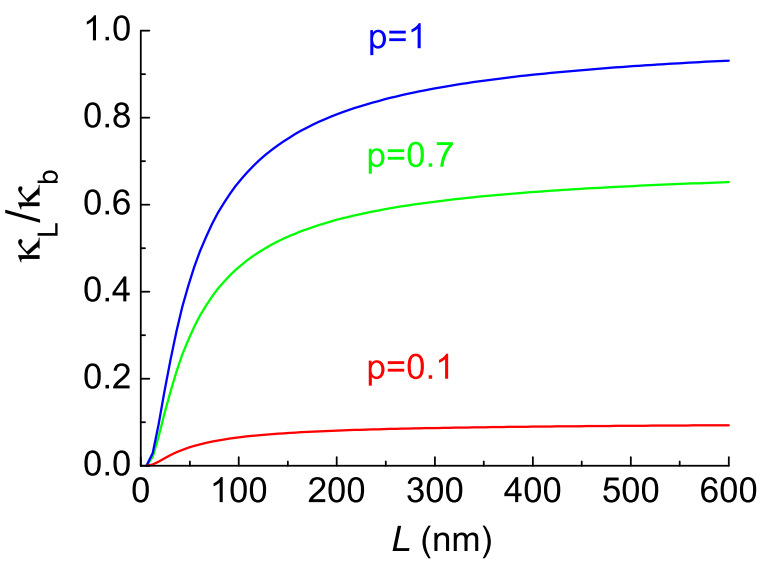

FIG. 1: Size-dependent dimensionless thermal conductivity for $\mathrm{Si}$ films in terms of Eq. (7), $\kappa_{L} / \kappa_{b}$ versus system size $L$ for $p=0.1$, 0.7 and 1, respectively. The other parameters in Eq. (7) are given in Table I.

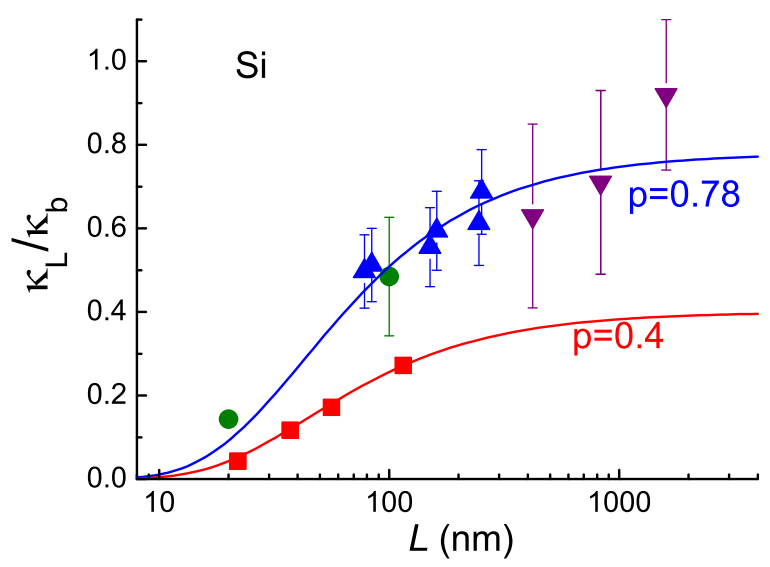

FIG. 2: Size-dependent thermal conductivity of Si nanowires and thin films at $300 \mathrm{~K}$. The symbols are experimental results, the uptriangles, circles and down-triangles for thin films (in-plane) cited from Refs.9,10 and 29, respectively, the squares for nanowires (along the axis) $\stackrel{8}{-}$ The curves are predictions from Eq. 7), the upper one for thin films with $p=0.78$, the lower one for nanowires with $p=0.4$.

asymptotic limits to be satisfied by Eq. (7): $L \rightarrow \infty$ and $p \rightarrow 1, \kappa_{L} \rightarrow \kappa_{b} ; L \rightarrow L_{0}$ or $p \rightarrow 0, \kappa_{L} \rightarrow 0$. In Fig. 1] we show $\kappa_{L} / \kappa_{b}$ versus system size $L$ for different roughness parameter $p$. The thermal conductivity spans a wide range, in which our prediction at $p=0.7$ for $L<100 \mathrm{~nm}$ is consistent with the previous theoretical prediction ${ }^{21}$ The intrinsic size effect decreases the conductivity obviously when the size is smaller than about $100 \mathrm{~nm}$. The surface effect related to $p$ can explain why the conductivity is low even in the large size in some case.

Figure 2 shows the size-dependent thermal conductivity of Si nanowires and thin films from the experiments and the comparison with our theoretical predictions from Eq. (7) with suit- able parameters $p$. The predictions are in good agreements with the experimental results. For carefully prepared Si films with thickness of $70-250 \mathrm{~nm}, \frac{9}{p} p=0.78$ corresponds with the experimental results, this large value of $p$ implies small diffusive scattering contribution due to the smooth surface related with the careful preparation of the nanostructures. For $\mathrm{Si}$ nanowires with diameter of $20-100 \mathrm{~nm}, \stackrel{8}{=} p=0.4$, reflecting the larger surface roughness of the nanowires in the sample fabrication.

Now we turn to the underlying physical meaning of the roughness parameter $p$. For the Si films with the thickness $L=100 \mathrm{~nm}$, if the surface roughness $\eta$, the mean root square deviation of height of the surface from the reference plane, is $2.2 \mathrm{~nm}$, which may be the case according to the precise fabrication technology of monocrystalline Si layers with thickness dispersion smaller than $4 \mathrm{~nm},{ }^{30} p=0.78$ can be obtained by a relation $p=1-10 \eta / L$; according to this relation, if $p=0.4, \eta$ is $1.32 \mathrm{~nm}$ for $L=22 \mathrm{~nm}$, which agrees with the experimental observation for the Si nanowires well,$\stackrel{8}{a}$ thus, this fitted formula is valid and can be used to determine $p$ by measuring or estimating $\eta$ of samples. The larger $\eta$ for a given $L$ corresponds to the smaller $p$, i.e., the rougher surface corresponds to the higher probability of diffusive scattering. Therefore, carefully fabricated structures with smoother surface will bring forth a larger conductivity under the same other conditions.

Figure 2 also shows that $p=0.78$ is applicable for the films spanning the size range of $20-100 \mathrm{~nm}, 70-250 \mathrm{~nm}$ and $400-1600 \mathrm{~nm}$, since the decreased $\eta$ can be achieved with decreasing $L$. Note that the surface roughness may be different for samples of different size even in the same experiment, but it can be controlled to be fewer than tens of nanometers generally in modern fabrication. ${ }^{30}$

Figure 3 shows the thermal conductivity of GaAs nanowires and thin films. The theoretical predictions with corresponding $p$ also show agreements with the experimental results from different groups. Similarly to Si nanowires, $p=0.07$ is also small for GaAs nanowires,$\frac{7}{\underline{\underline{T}}} \kappa_{L}<0.1 \kappa_{b}$. It seems that for nanowires, the thermal conduction is much worse compared to that of the corresponding films, which may be attributed to the restrained dimension, the surface fabrication precision and thus the increased surface scattering. For the superlattices with thickness of $140 \mathrm{~nm}, 31=0.98, \kappa_{L} \approx 0.9 \kappa_{b}$ is very high. For the superlattices of $10-100 \mathrm{~nm},{ }^{32} p=0.75$ and $\kappa_{L}$ is lower. The smaller $L$ corresponds to the relatively larger surface roughness and the smaller $p$, thus the lower conductivity. For the superlattices of $5-45 \mathrm{~nm}, \frac{33}{3} p=0.35, \kappa_{L}$ corresponds to the cross-plane conductivity measured and is even lower. Note that this low conductivity results also from the scattering contributions of the multi-interface corresponding to the increased interface roughness $\eta$, which has the weaker effect on the in-plane conductivity. When the size decreases to about $5 \mathrm{~nm}$, the structure and energy state of these nanosolids may be different from that of the corresponding crystals, the small size and large surface effect may not be sufficient to describe the physical properties of such small size structures and the quantum effect may be dominant, the present model is not suitable for that case. Some molecular dynamic calculation 


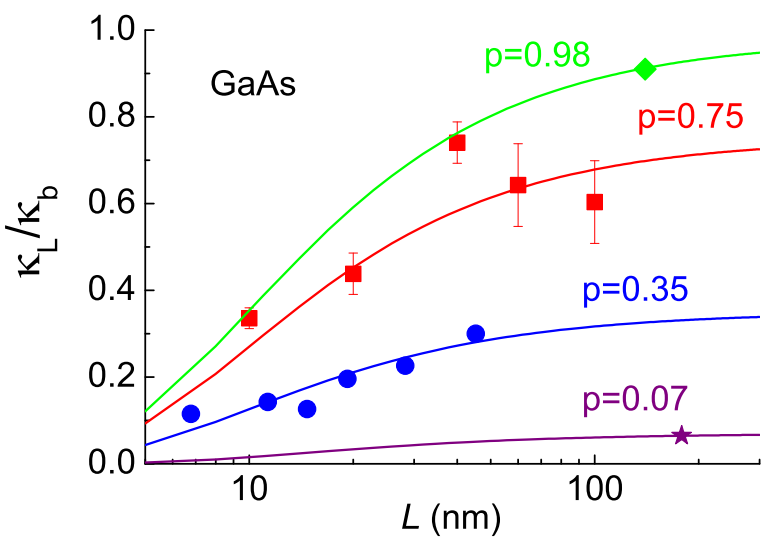

FIG. 3: Size-dependent thermal conductivity of GaAs nanowires and thin films. The star is experimental result of nanowire with diameter about $180 \mathrm{~nm}$ (corresponding to the rectangle cross-section with the same area) at $40 \mathrm{~K}, \frac{7}{-}$ considering the similar conductivity difference between the nanowire and the bulk extrapolated at $300 \mathrm{~K}$, the curve is prediction from Eq. (7) with $p=0.07$. The diamond is experimental result of GaAs/AlAs superlattice with thickness $140 \mathrm{~nm}, \stackrel{31}{3}$ assumed as the conductivity of a single GaAs film with the same thickness considering the similar structures between GaAs and AlAs (the following is the same), the curve for prediction with $p=0.98$. The squares are experimental results of GaAs/AlAs superlattices with thickness $10-100 \mathrm{~nm}$ (in-plane), ${ }^{32}$ the curve for prediction with $p=0.75$. The circles are experimental results of GaAs/AlAs superlattices with thickness 5-45 nm (cross-plane), ${ }^{33}$ the curve for prediction with $p=0.35$. shows the minimum with the size in the cross-plane thermal conductivity for the thinner superlattices without roughness, which is also theoretically attributed to a crossover between the particle transport and the wave transport ${ }^{34}$

In summary, we have derived a quantitative formula for the size-dependent thermal conductivity of nanoscale semiconducting systems by taking into account the intrinsic size effect and by correcting the bulk expression. The formula relates the thermal conductivity with the system size by the surface roughness parameter, the Knudsen number, the crystallinity length scale and the atomic vibration parameter. The model reveals the respective roles in the different size range of two basic effects on nanoscale thermal transport, i.e., the intrinsic size effect is predominant at the size of about 5-100 $\mathrm{nm}$, and the surface scattering effect is dominant at the larger size. The theory agrees well with the existing experimental results for $\mathrm{Si}$ and $\mathrm{GaAs}$ nanowires and thin films.

This work is supported in part by grants from the Faculty Research Grant of NUS and the DSTA Singapore under agreement DSTA POD0001821.
1 M. S. Gudiksen et al., Nature 415, 617 (2002); G. Chen and C. L. Tien, J. Appl. Phys. 74, 2167 (1993); K. E. Goodson and Y. S. Ju, Anuu. Rev. Mater. Sci. 29, 261 (1999).

${ }^{2}$ H. Kaburaki and M. Machida, Phys. Lett. A 181, 85 (1993); S. Lepri et al., Phys. Rev. Lett. 78, 1896 (1997); A. Fillipov et al., J. Phys. A 31, 7719 (1998); S. Lepri, Phys. Rev. E 58, 7165 (1998); K. Aoki and D. Kusnezov, Phys. Rev. Lett. 86, 4029 (2001); A. Pereverzev, Phys. Rev. E 68, 056124 (2003).

3 B. Hu, B. Li, and H. Zhao, Phys. Rev. E 57, 2992 (1998); A. V. Savin and O. V. Gendelman, Phys. Rev. E 67, 041205 (2003); B. Hu, B. Li, and H. Zhao, Phys. Rev. E 61, 3828 (2000); K. Aoki and D. Kusnezov, Phys. Lett. A 265, 250 (2000).

4 J.-S Wang and B. Li, Phys. Rev. Lett. 92, 074302 (2004); Phys. Rev. E 70, 021204 (2004).

${ }^{5}$ B. Li and J. Wang, Phys. Rev. Lett. 91 , 044301 (2003); B. Li et al., Chaos 15, 015121 (2005).

${ }^{6}$ C. Q. Sun et al., Phys. Rev. B 72, 134301 (2005).

${ }^{7}$ W. Fon et al., Phys. Rew. B 66, 045302 (2002).

8 D. Li et al., Appl. Phys. Lett. 83, 2934 (2003).

9 D. G. Cahill et al., J. Appl. Phys. 93, 793 (2003).

${ }_{11}$ W. Liu and M. Asheghi, Appl. Phys. Lett. 84, 3819 (2004).

11 J. M. Zimann, Electrons and Phonons (Oxford, Clarendon Press, 1960), p.288, p.58, p.296, p.456.

12 E. J. Post, Can. J. Phys. 31, 112 (1953).

13 A. R. Regel and V. M. Glazov, Semiconductors 29, 405 (1995).

14 A. Frenkel et al., Phys. Rev. B 48, 1283 (1993).

15 F. A. Lindemann, Z. Phys. 11, 609 (1910).

16 J. G. Dash, Rev. Mod. Phys. 71, 1737 (1999).
17 G. Soyez et al., Appl. Phys. Lett. 77, 1155 (2000).

18 F. G. Shi, J. Mater. Res. 9, 1307 (1994); Q. Jiang, H. X. Shi, and M. Zhao, J. Chem. Phys. 111, 2176 (1999); L. H. Liang et al., Phys. Rev. B 70, 205419 (2004).

19 N. F. Mott, Proc. R. Soc. London, Ser. A 146, 465 (1934).

20 J. R. Childress et al., Phys. Rev. B 44, 11689 (1991).

${ }^{21}$ E. Ziambaras and P. Hyldgaard, cond-mat/0503701

${ }^{22}$ Z. Zhang, M. Zhang, and Q. Jiang, Semi. Sci. Tech. 16, L33 (2001).

23 Sargent-Welch Scientific Company, Periodic Table of the Elements (Skokie, Illinois, 1980), p. 1.

${ }^{24}$ H. W. King, Physical Metallurgy, Ed. R. W. Cahn (North-Holland Pub. Co., Amsterdam, 1983), p. 64.

25 J. Zou and A. Balandin, J. Appl. Phys. 89, 2932 (2001).

${ }^{26}$ M. Hillert and L. I. Staffansson, Acta Chem. Scand. 24, 3618 (1970).

27 A. Ohtake et al., Appl. Surf. Sci. 212-213, 146 (2003).

28 S. M. Sze, Physics of Semiconductor Devices, 2nd Ed. (New York: Wiley, 1981), p. 850.

${ }^{29}$ M. Asheghi et al., Appl. Phys. Lett. 71, 1798 (1997).

30 M. Bruel, Nucl. Instrum. Meth. in Phys. Res. B 108, 313 (1996).

31 X. Y. Yu et al., Appl. Phys. Lett. 67, 3554 (1995).

${ }^{32}$ T. Yao, Appl. Phys. Lett. 51, 1798 (1987).

33 W. S. Capinski et al., Phys. Rev. B 59, 8105 (1999).

${ }^{34}$ M. V. Simkin and G. D. Mahan, Phys. Rev. Lett. 84, 927 (2000); B. C. Daly et al., Phys. Rev. B 66, 024301 (2002). 